\title{
Spectrum, Intensity and Coherence in Weighted Networks of a Financial Market
}

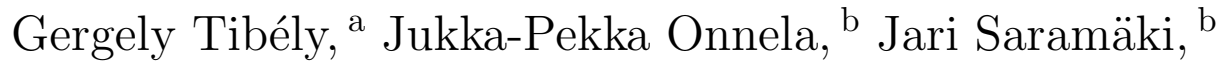 \\ Kimmo Kaski, ${ }^{b}$ János Kertész ${ }^{\text {a,b }}$ \\ ${ }^{a}$ Department of Theoretical Physics, Budapest University of Technology and \\ Economics, Budafoki t 8, H-1111 Budapest, Hungary \\ ${ }^{\mathrm{b}}$ Laboratory Computational Engineering, Helsinki University of Technology, \\ P.O.Box 9203, FIN-02015 HUT
}

\begin{abstract}
We construct a correlation matrix based financial network for a set of New York Stock Exchange (NYSE) traded stocks with stocks corresponding to nodes and the links between them added one after the other, according to the strength of the correlation between the nodes. The eigenvalue spectrum of the correlation matrix reflects the structure of the market, which also shows in the cluster structure of the emergent network. The stronger and more compact a cluster is, the earlier the eigenvalue representing the corresponding business sector occurs in the spectrum. On the other hand, if groups of stocks belonging to a given business sector are considered as a fully connected subgraph of the final network, their intensity and coherence can be monitored as a function of time. This approach indicates to what extent the business sector classifications are visible in market prices, which in turn enables us to gauge the extent of group-behaviour exhibited by stocks belonging to a given business sector.
\end{abstract}

\section{Introduction}

In the world of business, companies interact with one another, creating an evolving complex system (1). While the details of these interactions are not known, as far as price changes are concerned, they are reflected in the correlations of stock prices. Correlations are central in investment theory and risk management, and also serve as inputs to the portfolio optimisation problem in the classical Markowitz portfolio theory (2).

Complex networks can be seen to provide a general framework for studying systems with large numbers of interacting agents (3). The nodes of the network 
represent the agents and a link connecting two nodes indicates an interaction between them. In this framework, interactions have typically been considered binary in nature, meaning that two nodes are either connected or not. However, in a system with correlated nodes the notion of binary interactions implies setting a threshold value for interaction strength, above which the link exists and below it does not. This entails a certain loss of information as for the properties of the system, which can be circumvented by assigning weights on the links to reflect their interaction strengths. These are naturally identified by the corresponding elements of the correlation matrix.

In this paper we study a financial network in which the nodes correspond to stocks and links to return correlation based interactions between them. Mantegna (4) was the first to construct such networks and the idea was then followed and extended by others $(\underline{5} ; 6 ;$; $;$; $;$; $)$.

\section{Network Construction}

We start by considering a price time series for a set of $N$ stocks and denote the daily closing price of stock $i$ at time $\tau$ (an actual date) by $P_{i}(\tau)$. Here we will analyse $N=116$ NYSE-traded stocks from the S\&P 500 index over the period from 1.1.1982 to 31.12.2000, consisting the total of 4787 daily closing price quotes for each stock. As it is common among investors in the financial market, we will work in terms of relative returns defined as $r_{i}(\tau)=\ln P_{i}(\tau)-$ $\ln P_{i}(\tau-1)$. For the purpose of smoothening, we set a moving time window of width $T$, here $T=1000$ trading days $(\approx 4$ years, for 250 trading days a year), and obtain a return vector $\mathbf{r}_{i}^{t}$ for stock $i$, where the superscript $t$ enumerates the time window under consideration. Now the equal time correlation coefficients between assets $i$ and $j$ can be written as follows

$$
\rho_{i j}^{t}=\frac{\left\langle\mathbf{r}_{i}^{t} \mathbf{r}_{j}^{t}\right\rangle-\left\langle\mathbf{r}_{i}^{t}\right\rangle\left\langle\mathbf{r}_{j}^{t}\right\rangle}{\sqrt{\left[\left\langle\mathbf{r}_{i}^{t^{2}}\right\rangle-\left\langle\mathbf{r}_{i}^{t}\right\rangle^{2}\right]\left[\left\langle\mathbf{r}_{j}^{t^{2}}\right\rangle-\left\langle\mathbf{r}_{j}^{t}\right\rangle^{2}\right]}},
$$

where $\langle\ldots\rangle$ indicates a time average over the consecutive trading days included in the return vectors. These correlation coefficients between $N$ assets form a symmetric $N \times N$ correlation matrix $\mathbf{C}^{t}$ with elements $\rho_{i j}^{t}$. The time windows are displaced by $\delta T$, where we have used a step size of one trading week, i.e. $\delta T=5$ days.

We construct the network first by ranking the interaction strengths $w_{i j}$ taken as absolute values of the correlation coefficients. Due to the fact that $\rho_{i j}^{t}$ vary between -1 and 1 , the interaction strengths $w_{i j}^{t}=\left|\rho_{i j}^{t}\right|$ are limited to the $[0,1]$ interval. Then the network is constructed such that the links are added one 
after the other, starting from the strongest one according to the ranking. The emergent network is characterized by a parameter $p$, namely the ratio of the created links to the number of all possible links, $N(N-1) / 2$. In the end of the procedure when $p=1$, we have a fully connected weighted network. In (10) we have reported this approach and found clear evidence of strong intra-business sector clustering for low values of $p$, where we followed the Forbes business sector labelling of stocks into 12 categories, such as Energy and Utilities (11).

\section{Spectral Properties}

The spectra of financial correlation matrices have been studied in detail, producing interesting results (12). The eigenvalues can be classified as follows: i) There is a quasi-continuum of small eigenvalues which can be well described by the random matrix theory corresponding to noise, and the majority of them fall into this category. ii) The largest eigenvalue is far from the rest and it corresponds to the global behaviour of the market. iii) The discrete spectrum of intermediate eigenvalues carries important information about the correlations that can be related to market taxonomy. As an example of applications, the eigenvalue spectrum can be used to denoise the correlation matrix (13). The eigenvalue spectrum also reflects the business sector structure of the network. Therefore, it is natural to ask the question: How do the typical eigenvalues emerge as a function of the ratio of the links present or occupation $p$ ?

Here we calculate the eigenvalue spectrum of the matrix $w_{i j}-\delta_{i j}$ for different values of $p$, where $\delta_{i j}$ is the Kronecker delta function. Starting from the strongest links first the most correlated parts emerge in the network. They form separated clusters with high clustering coefficients, thus the emerging structure is far from random. The eigenvalue spectrum reflects this property. Already for very small values of $p$ the largest eigenvalue separates from the rest and the components are quite uniformly distributed among the stocks already included in the network, indicating the dominance of the global market behaviour. To give the eigenvalues some physical meaning it is convenient to plot the values of each of the 116 components of the eigenvector corresponding to the chosen eigenvalue.

For small $p$ the next largest eigenvalues have a clear meaning: They correspond to highly correlated business sectors which emerge first in the network as isolated clusters. These eigenvalues are inherited to the $p=1$ case (though their ranking can change). Fig. 1 shows two such eigenvalues; for the fully connected graph $(p=1)$ and for low occupation, $p=0.024$. This value of $p$ corresponds to 160 links in the network at which stage its structure is shown in Fig. 2. We can conclude that the visually well-separated clusters for low occupation $p$ induces a large eigenvalue in the category iii) which is inherited 

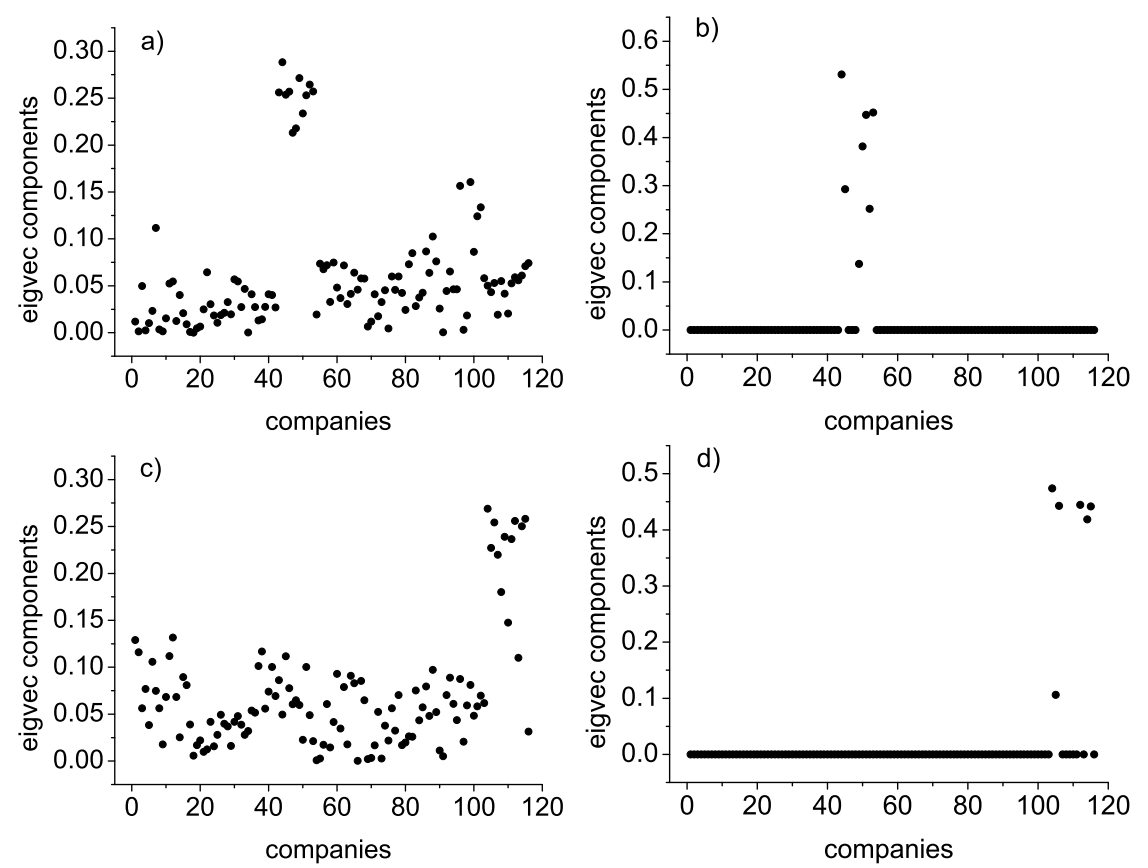

Fig. 1. Eigenvector components for the Energy sector a) for $p=1$, and $\mathrm{b}$ ) for $p=0.024$, and for the Utilities sector c) for $p=1$, and d) for $p=0.024$.

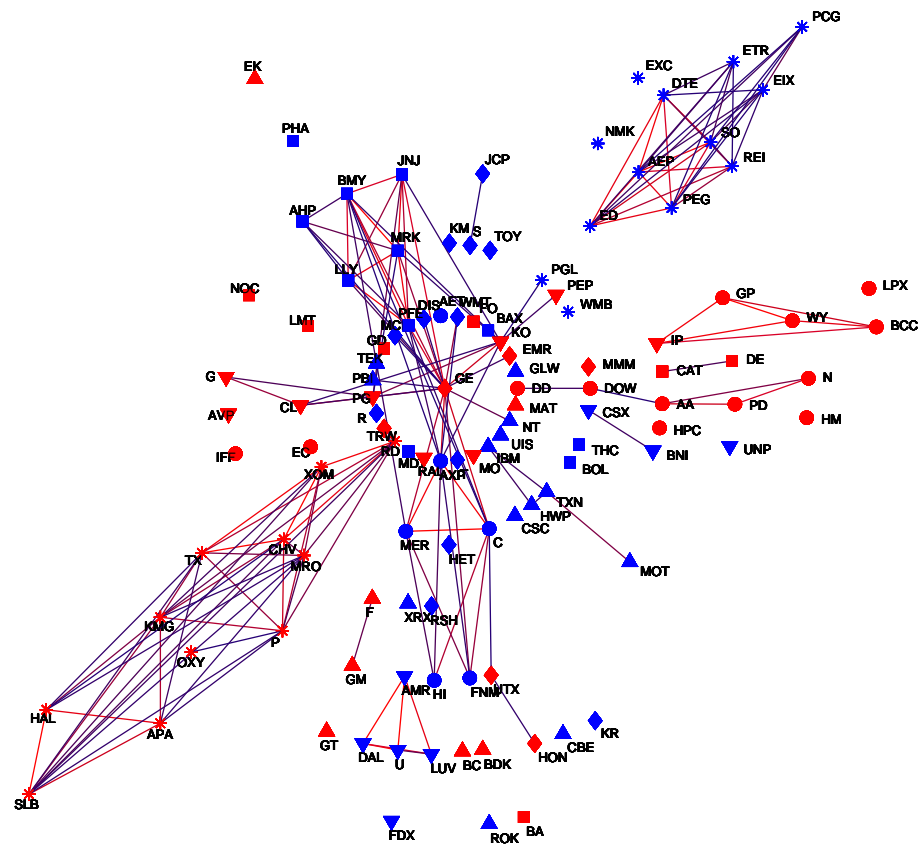

Fig. 2. The network for occupation $p=0.024$. The Energy sector is pointing to South-West and the Utilities sector to North-East direction. Different symbols correspond to different business sectors according to Forbes classification.

to the final fully connected case. 


\section{Subgraph Intensity and Coherence}

In order to study the clustering properties in more detail, let us consider any cluster or subgraph $g$ in these networks by defining two additional measures. To characterise how compact or tight the subgraph is, we use the concept of subgraph intensity $I(g)$ introduced earlier in (14). This measure allows us to characterise the interaction patterns within clusters. By denoting $v_{g}$ the set of nodes and $\ell_{g}$ the set of links in the subgraph with weights $w_{i j}$, we can express subgraph intensity as the geometric mean of its weights as

$$
I(g)=\left(\prod_{(i j) \in \ell_{g}} w_{i j}\right)^{1 /\left|\ell_{g}\right|} .
$$

However, with this definition the subgraph intensity $I(g)$ may turn out to be low because one of the weights is very low, or all the weights are low. In order to distinguish between these two extremes, we use the concept of subgraph coherence $Q(g)$ introduced in (14) and defined as the ratio of the geometric mean to the arithmetic mean of the weights:

$$
Q(g)=I\left|\ell_{g}\right| / \sum_{(i j) \in \ell_{g}} w_{i j} .
$$

This coherence measure gets values in the $[0,1]$ interval and is close to unity only if the subgraph weights do not differ much, i.e. they are internally coherent. To compare the intensity and coherence values of various clusters, we establish a reference, consisting the entire market. In other words, we take all the $N$ nodes and $N(N-1) / 2$ links making up the entire network $G$, and then with Eqs. 2 and 3 calculate $I(G)$ and $Q(G)$, respectively. Here we will use the relative quantities, i.e. relative cluster intensity for cluster $g$, given by $I(g) / I(G)$, and relative cluster coherence, given by $Q(g) / Q(G)$.

We will apply these measures to the same set of 116 NYSE-traded stocks from the S\&P 500 index and devide the stocks into clusters by using the same business sector labels for each stocks as above (11). Given these labels for each stock, we determine the subgraph intensity and coherence to gauge how stocks belonging to a given business sector behave as a function of time. Now let us consider a cluster $g_{n}$, constructed such that all of its nodes $v_{g}$ belong to the same business sector and $n$ denotes the number of nodes in this cluster. Then we add all the $n(n-1) / 2$ links corresponding to the interaction strengths between nodes within $g_{n}$. In one extreme, if all the link weights are equal to unity, every node in $g_{n}$ interacts maximally with its $n-1$ neighbours. In the other extreme, if one or more of the weights are zero, the subgraph intensity for the fully connected subgraph $g_{n}$ tends to zero because the original topological 

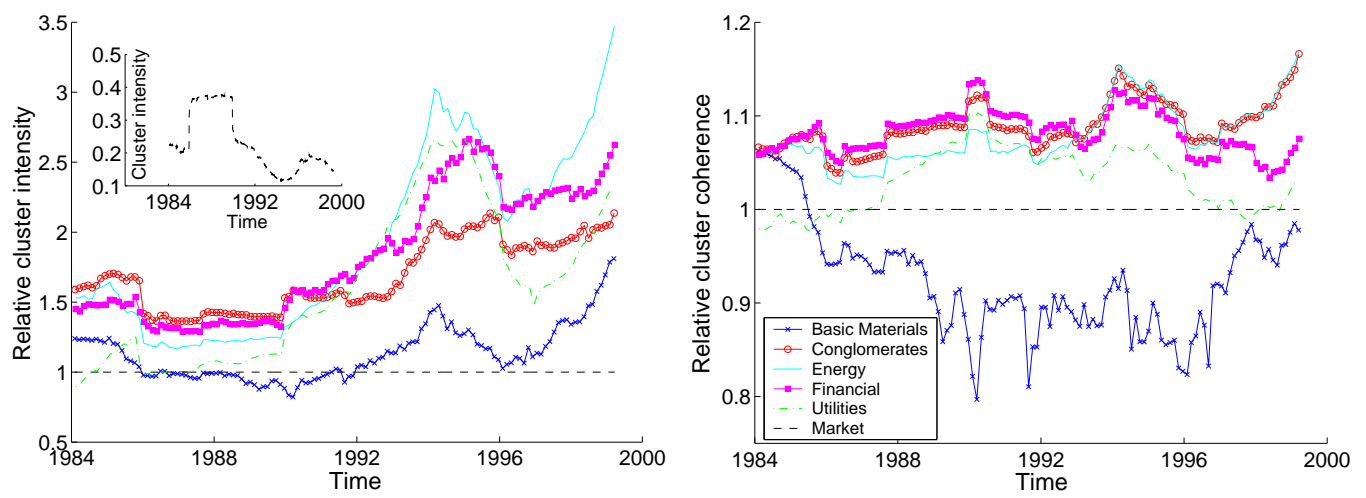

Fig. 3. (a) Relative cluster intensity as a function of time for select clusters. In the inset: The absolute cluster intensity for the whole market used for normalisation. (b) Relative cluster coherence as a function of time.

structure no longer exists. While this may seem extreme, it is important to realize that the companies of any given business sector are expected to interact, at least to some extent, with all other companies within the sector. In practise, however, it rarely happens that we would have a weight $w_{i j}=0$ exactly.

In Fig. 3(a) we show the relative cluster intensity as a function of time for selected business sector clusters. Here the values above unity indicate that the intensity of the cluster is higher than that of the market. This implies that in most cases stocks belonging to a given business sector are tied together in the sense that intra-cluster interaction strengths are considerably stronger than the whole market interaction. In the inset of Fig. 3(a) we have depicted the absolute cluster intensity for the whole market, which shows high values roughly between 1986 and 1990. This is caused by stock market crash (Black Monday, 1987) when the market behaves in a unified manner. It should be noted here that although the crash is a localized event, in our analysis it covers an extended period due to the moving window length being four years. From Fig. 3(a) we also see that the crash compresses the relative cluster intensities, which means that the cluster-specific behaviour is temporarily suppressed by the crash. After the market recovers, the clusters regain their normal structural features $(15)$.

In Fig. 3(b) we show the relative coherence as a function of time for selected business sector clusters. All clusters except Basic Materials turn out to be more coherent than the market. One possible explanation is that for Basic Materials the industry classification scheme is too course, because in finer classification this sector includes stocks diversely from Metal Mining, Paper, Gold \& Silver and Forestry \& Wood Products. Consequently, it is not that surprising that the cluster intensity remains low, at times even falling below the market reference. Similarly, the low coherence values indicate that there are stocks in this cluster with very high correlations due to those belonging to the same industry, such as gold mining, but also very low due to companies 
belonging to different industries. In conclusion, our results indicate that, in most cases, stocks belonging to the same business sector have higher intensity and more coherent intra-cluster than inter-cluster interactions.

Support from the Academy of Finland (Center of Excellence programme), OTKA T049238 and COST P10 is acknowledged.

\section{References}

[1] W. B. Arthur, S. N. Durlauf and D. A. Lane (eds.), The economy as an evolving complex system II, Addison-Wesley, Reading, Massachusetts (1997).

[2] H. M. Markowitz, Journal of Finance 7, 77 (1952).

[3] R. Albert, A.-L. Barabasi, Reviews of Modern Physics 74, 47 (2002).

[4] R. N. Mantegna, European Physical Journal B 11, 193 (1999).

[5] N. Vandewalle, F. Brisbois, X. Tordoir, Quantitative Finance 1, 372 (2001).

[6] M. Marsili, Quantitative Finance 2, 297-302 (2002).

[7] G. Caldarelli, S. Battiston, D. Garlaschelli, M. Catanzaro, in: E. Ben-Naim, H. Frauenfelder, Z. Toroczkai (eds) Complex Networks. Springer (2004).

[8] J.-P. Onnela, A. Chakraborti, K.Kaski, J. Kertész, A. Kanto, Physical Review E 68, 056110 (2003), J.-P. Onnela, A. Chakraborti, K. Kaski, J. Kertész, A. Kanto, Physica Scripta T106, 48-54 (2003).

[9] T. Aste , T. Di Matteo, M. Tumminello, R. N. Mantegna in: Noise and Fluctuations in Econophysics and Finance, Edited by D. Abbott, J.-P. Bouchaud, X. Gabaix, J. L. McCauley, Proc. of SPIE, Vol. 5848 (SPIE, Bellingham, WA, 2005) p. 100, T. Di Matteo, T. Aste, R. N. Mantegna, Physica A 339, 181, (2004)

[10] J.-P. Onnela, K. Kaski, J. Kertész, European Physical Journal B 38, 353 (2003).

[11] Forbes web-site: www.forbes.com.

[12] L. Laloux, P. Cizeau, J.-P. Bouchaud and M. Potters, Phys. Rev. Lett. 83, 1467 (1999), V. Plerou, P. Gopikrishnan, B. Rosenow, L. A. N. Amaral, and H. E. Stanley Phys. Rev. Lett. 83, 1471 (1999)

[13] G. Papp, S. Pafka, M. A. Nowak, I. Kondor Acta Physica Polonica 36, $2757(2005)$

[14] J.-P. Onnela, J. Saramäki, J. Kertész, K. Kaski, Physical Review E 71, 065103 (2005).

[15] J.-P. Onnela, J. Saramäki, K. Kaski, J. Kertész, in: Takayasu H (ed.) Practical Fruits of Econophysics. Springer (2006), p. 302. 